\title{
Self replicating systems
}

\author{
Volker Patzke and Günter von Kiedrowski* \\ Institute for Organic Chemistry, Ruhr-University, Bochum, Universitätsstraße 150, \\ 44801 Bochum, Germany \\ E-mail: kiedro@rub.de
}

Dedicated to Prof. Dr. L.-F. Tietze on the occasion of his 65th anniversary

\begin{abstract}
Chemical self-replicating systems have been the subject of various investigations over the last 20 years. This mini review outlines the design of minimal model systems, mainly based on oligonucleotides and on peptides and artificial compounds, which implement template-directed reactions. In order to examine the reaction mechanism and to attain an exponential replication the development of self-replicating systems is pointed out.
\end{abstract}

Keywords: Self-replication, template-directed reactions, reaction mechanism, oligonucleotides, autocatalysis, cross-catalysis

\section{Contents}

1. Nucleic acid replicators

1.1. Polycondensations of activated mononucleotides directed by templates

1.2 From chemical ligation to self-replication

1.3 Competition and cooperation in non-enzymatic self-replicating systems

2. Replication in non-nucleotidic model systems

3. Outlook: toward exponential replication

\section{Introduction}

The theory of Darwinian evolution describes the origin of biological information. In general, an evolving system (i.e., an information-gaining system) is able to metabolize, to self-replicate, and to undergo mutations (as was stated by Oparin in 1924). ${ }^{1}$ Thus self-replication is one of the three criteria that enable us to distinguish non-living from living systems. Since nucleic acids carry the 
inherent ability for complementary base-pairing (and replication), they are candidates to have been the first reproducing molecules. Kühn ${ }^{2}$ and others (Crick, ${ }^{3} \mathrm{Orgel}^{4}{ }^{4}$ Eigen and Schuster, $1979 ;{ }^{5}$ Kühn and Waser, $1981 ;^{6}$ for a review, see Joyce ${ }^{7}$ ) have drawn the picture of an RNA world that might have existed before translation was invented. This picture was supported by the finding RNA (and also DNA) that can act as an enzyme-like catalyst (Joyce, ${ }^{7}$ Sharp, ${ }^{8}$ Cech, ${ }^{9}$ Breaker and Joyce ${ }^{10}$ ) or even as a, "self-replicating ligase ribozyme" (Paul and Joyce). ${ }^{11}$

A simple three-step model can be used to conceptualize the process of molecular template directed replication (Figure 1). In this model, the template molecule $\mathbf{T}$ is self-complementary and thus able to autocatalytically augment itself. In the first step, the template $\mathbf{T}$ reversibly binds its constituents $\mathbf{A}$ and $\mathbf{B}$ to yield a termolecular complex $\mathbf{M}$. Within this complex, the reactive ends of the precursors are held in close proximity, which facilitates the formation of a covalent bond between them.

In the following step, the termolecular complex $\mathbf{M}$ is irreversibly transformed into the duplex D. Reversible dissociation of $\mathbf{D}$ gives two template molecules, each of which can initiate a new replication cycle. The minimal representation given in Figure 1 has served as a successful aid for the development of non-enzymatic self-replicating systems based on nucleotidic and nonnucleotidic precursors, as will be shown.

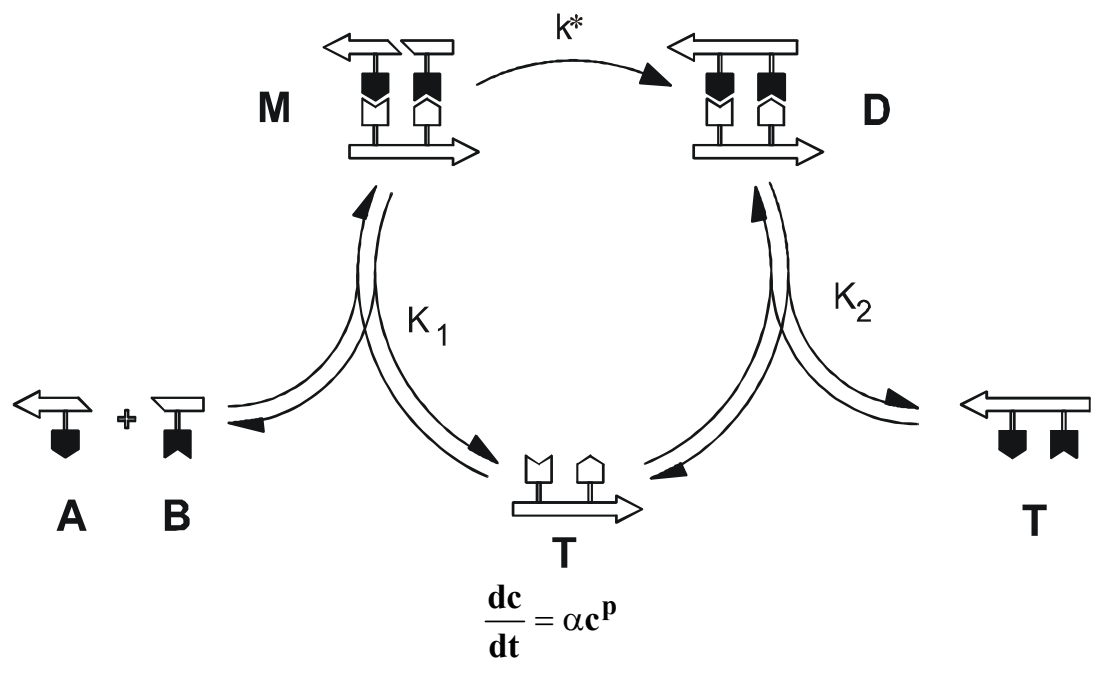

a) parabolic: $\mathbf{p}=\frac{1}{2}, \mathbf{c}_{\mathbf{t}}=\left(\mathbf{c}_{0}{ }^{1 / 2}+\alpha \frac{\mathbf{t}}{2}\right)^{2}$

b) exponential: $\mathbf{p}=1, \mathbf{c}_{\mathbf{t}}=\mathbf{c}_{0} \mathbf{e}^{\alpha \mathbf{t}}$

Figure 1. Schematic representation of a self-replicating system; rate equations for parabolic and exponential growth.

Chemical self-replicating systems have been designed in order to identify the minimal requirements for molecular replication, to translate the principle into synthetic supramolecular 
systems and to derive a better understanding of the scope and limitation of self-organization processes that are believed to be relevant to the origin of life on earth. Current implementations make use of oligonucleotide analogues, peptides, and other molecules as templates and are based either on autocatalytic, cross-catalytic, or collectively catalytic pathways for template formation. A common problem of these systems is product inhibition leading to parabolic instead of exponential amplification. The latter is the dynamic prerequisite for selection in the Darwinian sense. $^{12}$

\section{Nucleic acid replicators}

\subsection{Polycondensations of activated mononucleotides directed by template}

The template-directed synthesis of oligonucleotides from activated mononucleotide precursors (such as monoribonucleoside-5'-phosphorimidazolides) has been studied extensively by Orgel and his co-workers since 1968 . $^{4,13,14}$ These studies revealed two basic principles:

1. The polycondensation yields two different regioisomeric products, one with a 2 '-5'- and one with the natural 3'-5'-linkage. The regioselectivity depends on various parameters (e.g., the template, nature of the leaving group, presence of metal ions, etc.).

2. The polycondensations were found to be rather efficient with a pyrimidine-rich template. In contrast, a purine-rich oligo- or polynucleotide acts as a poor template.

The findings concerning the templates were envisioned as a major obstacle for further attempts to realize self-replicating systems based on mononucleotide precursors. Despite these difficulties, the Orgel group succeeded in demonstrating the non-enzymatic template-directed synthesis of fully complementary products. Templates as long as 14- mers were successfully transcribed. $^{15}$ However, no complete replication cycle could be achieved when using mononucleotides as precursors.

\subsection{From chemical ligation to self-replication}

The work of Orgel and coworkers clearly demonstrated that transcription with information transfer can occur in the absence of enzymes. Due to the problems previously described it seemed worth while to use oligonucleotides as building material and to employ chemical ligations instead of polycondensations as coupling reactions. The first example of a chemical ligation, namely a template-directed condensation of activated oligonucleotides, was reported by Naylor et al. ${ }^{16}$ They demonstrated that the condensation of pentathymidylic acid and hexathymidylic acid molecules could be catalyzed by a poly(A) template in the presence of the water-soluble carbodiimide EDC (1-ethyl-3-(3-dimethylaminopropyl)-carbodiimide). Further examples of chemical ligation reactions have been reported by Shabarova's group in Moscow. ${ }^{17,18}$ A successful demonstration of enzyme-free nucleic acid replication based on an 
autocatalytic chemical system was reported by von Kiedrowski in $1986 .{ }^{19}$ A 5 '-terminally protected trideoxynucleotide 3'-phosphate d(Me-CCG-p) (1) and a complementary 3'-protected trideoxynucleotide d(CGG-p') (2) were reacted in the presence of EDC to yield the selfcomplementary hexadeoxynucleotide (Me-CCG-CGG-p') (3) with natural phosphodiester linkage as well as the $3^{\prime}-3^{\prime}$-linked pyrophosphate of $\mathbf{1}$. The sequences chosen were such that the product 3 could act as a template for its own production. The hexamer formation proceeds via a termolecular complex $\mathbf{C}$ formed from 1, 2, and $\mathbf{3}$, in which reactive ends are in close spatial proximity and thus ready to be ligated. During the course of reaction, the activated 3 '-phosphate of $\mathbf{1}$ is attacked by the adjacent 5'-hydroxyl group of $\mathbf{2}$, forming a 3'-5'-internucleotide bond between the trimers (see Figure 2).

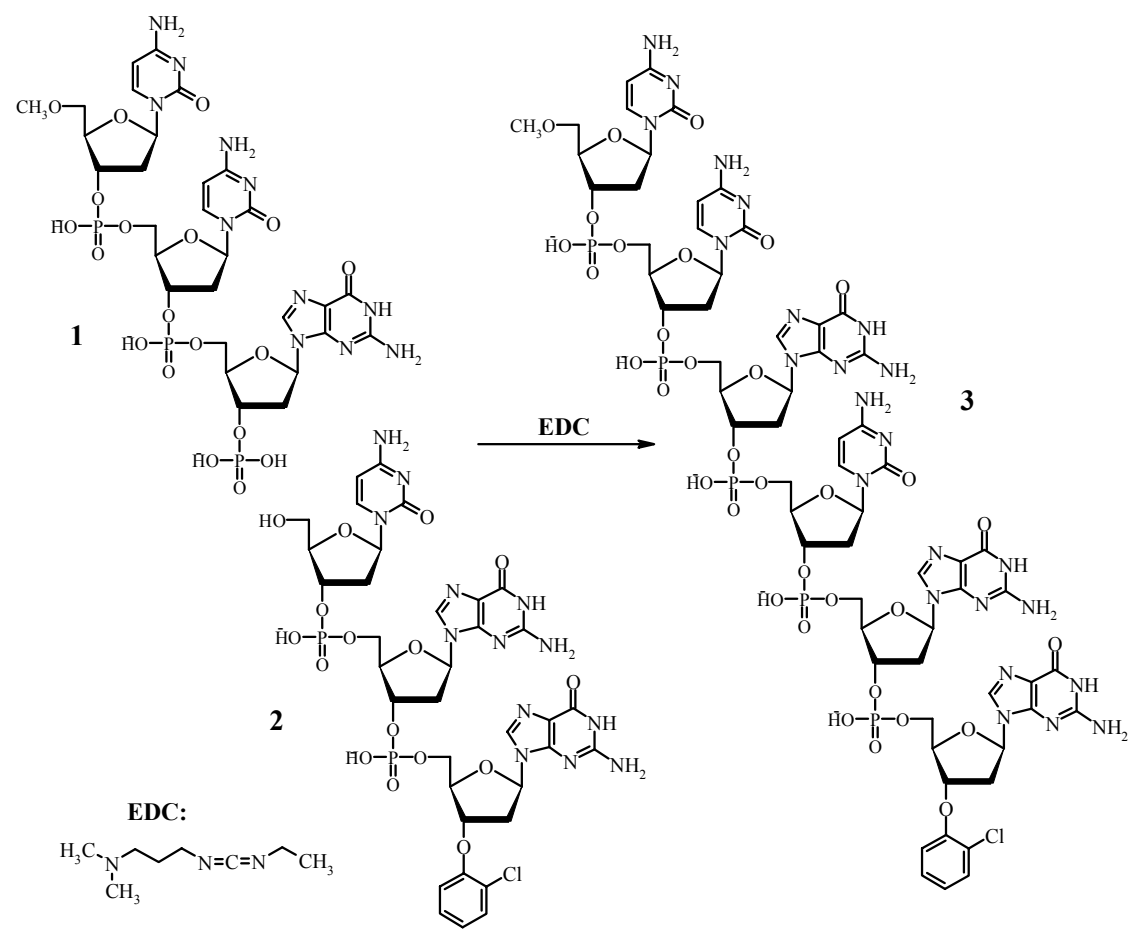

Figure 2. First non-enzymatic self-replicating system.

The resulting template duplex then can dissociate to yield two free template molecules each of which can initiate a new replication cycle. Two parallel pathways for the formation of hexameric templates exist, as can be shown by kinetic studies using HPLC. Both pathways, the template-dependent, autocatalytic pathway and the template-independent, non-autocatalytic pathway, contribute to product formation. The latter pathway has been found to be predominant. Moreover, the experiments revealed that the addition of template did not increase the rate of autocatalytic template formation in a linear sense. Instead, the initial rate of autocatalytic synthesis was found to be proportional to the square root of the template concentration (a finding that was termed the square root law of autocatalysis). Thus, the reaction order in this 
autocatalytic self-replicating system was found to be $1 / 2$ rather than 1 , a finding in contrast to most autocatalytic reactions known so far. According to theory, a square root law is expected in the previously described cases, in which most of the template molecules remain in their doublehelical (duplex) form, which leaves them in an "inactive" state. In other words, a square root reflects the influence both of autocatalysis and of product inhibition.

Another example of an autocatalytic system following the square root law was published by Zielinsky and Orgel in $1987 .{ }^{20}$ The diribonucleotide analogues $\left(\mathrm{G}_{\mathrm{NHp}} \mathrm{C}_{\mathrm{NH} 2}\right)(4)$ and $\left({ }_{\mathrm{p}} \mathrm{G}_{\mathrm{NHp}} \mathrm{C}_{\mathrm{N} 3}\right)$ (5) were ligated in the presence of water-soluble carbodiimide (EDC) and self-complementary tetraribonuclteotide triphosphoramidate $\left(\mathrm{G}_{\mathrm{NHp}} \mathrm{C}_{\mathrm{NHp}} \mathrm{G}_{\mathrm{NHp}} \mathrm{C}_{\mathrm{N} 3}\right)$ (6), serving as a template (see Figure 3). This was the first demonstration of self-replication of nucleic acid-like oligomers bearing an artificial backbone structure. In kinetic studies, the autocatalytic nature of template synthesis was obvious from the square-root dependence of the initial reaction rate on the template concentration. In theory, every true autocatalytic system should show a sigmoidal concentration-time profile. ${ }^{21}$ Due to the predominance of the non-autocatalytic pathway this was not true for either system just described. The autocatalytic nature of these was the first demonstration of self-replication of nucleic acid-like oligomers bearing an artificial backbone structure. In kinetic studies, the autocatalytic nature of template synthesis was obvious from the square root dependence of the initial reaction rate on the template systems was ascertained indirectly by observing the increase in the initial reaction rate when seeding the reaction mixtures with increasing amounts of template.
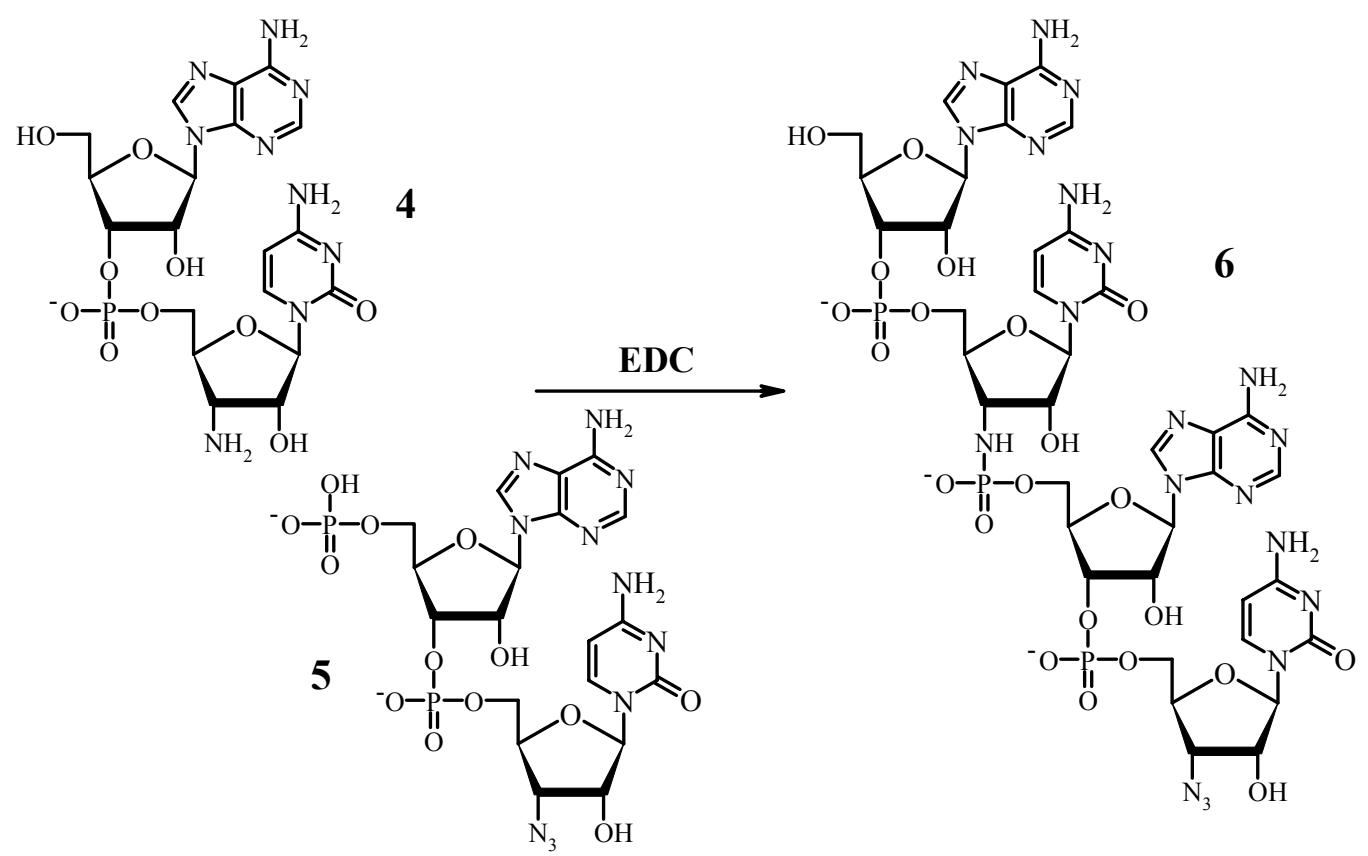

Figure 3. Self-replicating tetraribonucleotide according to Zielinski and Orgel. ${ }^{20}$ 
In the years of research following 1987, a major goal was to enhance the template-instructed autocatalytic synthesis while keeping the reaction rate of the non-instructed synthesis as low as possible. It can be shown that autocatalytic synthesis particularly benefits from an increased nucleophilicity of the attacking $5^{\prime}$-group. When trimer 2 was used in its 5 '-phosphorylated form instead of the 5'-hydroxyl form, the carbodiimide-dependent condensation with $\mathbf{1}$ yielded hexamers bearing a central 3'-5'-pyrophosphate dependent condensation with 1 yielded hexamers bearing a central 3'-5'-pyrophosphate linkage. Due to this modification, the rate of the templateinduced hexamer formation was increased by roughly two orders of magnitude. ${ }^{22}$ Replacing the 5'-phosphate by a 5'-amino group led to the formation of a 3'-5'-phosphoramidate bond and resulted in a rate enhancement of almost four orders of magnitude as compared to the phosphodiester system.

In addition to that, the autocatalytic synthesis of template molecules was found to be more selective in the case of the faster replicators. The quantity $e$ (a measure of the ratio of autocatalytic over background synthesis) could be increased from $16 \mathrm{M}^{-1 / 2}$ in the phosphodiester system to $430 \mathrm{M}^{-1 / 2}$ in the $3^{\prime}-5^{\prime}$-phosphoramidate system. The first direct evidence for a sigmoidal increase in template concentration was found in the latter system, by von Kiedrowski et al. ${ }^{23,24}$ Shortly after these observations, a second chemical self-replicating system was reported to ex hibit sigmoidal growth. ${ }^{25}$ A sigmoidal shape for template formation gives direct evidence of autocatalytic growth since this type of growth is a direct consequence of the square root law of autocatalysis. Following the square root law, the increase of template concentration at early reaction times is parabolic rather than exponential. For these early points in time, the integrated form of $\mathrm{dc} / \mathrm{dt}=\alpha \mathrm{c}^{1 / 2}$ ( $\alpha$ is an empirical constant) can be approximated using a second order polynomial of time whose graph shows a parabola. Parabolic growth is a direct consequence of the square root law in cases where non-autocatalytic synthesis is negligible. A detailed analytical treatment by von Kiedrowski ${ }^{21}$ revealed three types of autocatalytic growth as borderline cases. From theoretical considerations, it was also concluded that the autocatalytic growth order is solely dependent on the thermodynamics of the coupled equilibria, rather than on the energy on the transition state.

Further studies were devoted to the subject of sequence selectivity in the self-replication of hexadeoxynucleotides, giving further evidence for the autocatalytic nature of this reaction. ${ }^{26} \mathrm{~A}$ homologous set of trimer-3'-phosphates bearing the general sequence ${ }^{\mathrm{PG}} \mathrm{XYZp}$ (PG = protective group) was synthesized, where $\mathrm{X}, \mathrm{Y}$, and $\mathrm{Z}$ could represent either $\mathrm{C}$ or $\mathrm{G}$ monomers. Each of the trimer 3'-phosphates was reacted with the aminotrimer ${ }^{\mathrm{H} 2 \mathrm{~N}} \mathrm{CGG}^{\mathrm{PG}}$ in the presence of EDC. The trimer 3'-phosphate bearing the sequence CCG was formed significantly faster than all the other trimers. Moreover, addition of template CCGCGG, complementary to the ligated trimers, stimulated only the synthesis of the proper phosphoramidate while having a negligible influence on the variant sequences. These studies demonstrated that autocatalysis can occur only if the sequences of both trimers match the sequence of the resulting hexamer according to the WatsonCrick base-pairing rules. They also showed that the condensation reactions are controlled predominantly by the stacking of nucleobases flanking the newly formed internucleotide linkage. 
Hexamers bearing a central G-C sub-sequence, for example, are formed one order of magnitude faster than hexamers with a central $\mathrm{C}-\mathrm{G}$ sub-sequence. In general, the following reactivity order was found: $\mathrm{G}-\mathrm{G}>\mathrm{G}-\mathrm{C}>\mathrm{C}-\mathrm{G}>\mathrm{C}-\mathrm{C}$, a finding that is in good correspondence with experiments with non-autocatalytic chemical ligations. Further studies revealed a remarkable temperature dependence in hexadeoxynucleotide self-replication. Each parabolic replicator shows a rate optimum at a certain temperature, which was found to be close to the measurable melting temperature of the respective hexamer duplex. ${ }^{26}$ Again, this is in good correspondence with minimal replicator theory. Generally, the autocatalytic reaction rate is given by $\mathrm{k}[\mathrm{C}]$, where $\mathrm{k}$ equals the rate constant for irreversible internucleotide bond formation and $[\mathrm{C}]$ is the equilibrium concentration of the termolecular complex. Because the rate constant $\mathrm{k}$ increases with temperature (according to Arrhenius' law) and [C] decreases due to the melting of the termolecular complex, the reaction rate as a function of temperature is expected to pass a maximum at the temperature $t_{\text {opt }} \cdot t_{\text {opt }}$ itself depends on both the concentrations of template and its precursors as well as the thermodynamic stabilities of the termolecular complex and the template duplex.

\subsection{Competition and cooperation in non-enzymatic self-replicating systems}

The following studies in our laboratory were devoted to the question of information transfer in a more complex system, in which a number of alternative templates can be produced from a set of common precursors. Such a system was realized in an experiment in which the sequence CCGCGG was synthesized from three fragments. ${ }^{27}$ The trimer-3'-phosphate ${ }^{P G} \mathrm{CCGp}(\mathbf{A})$, the 5'aminodimer-3'-phosphate ${ }^{\mathrm{H} 2 \mathrm{~N}} \mathrm{CGp}(\mathbf{B})$ and the $5^{\prime}$-aminomonomer ${ }^{\mathrm{H} 2 \mathrm{~N}} \mathrm{G}(\mathbf{C})$ were allowed to react in the presence of 1-methylimidazole (MeIm) and EDC. The five products $\mathbf{A B}, \mathbf{A C}, \mathbf{B C}, \mathbf{B B}$, and ABC, all bearing central 3'-5'-phosphoramidate linkages, could be identified. To monitor the whole system using HPLC kinetic analysis, it was necessary to reduce its complexity. In order to detect possible catalytic-, cross-catalytic-, or autocatalytic- pathways (couplings) induced by the different products, the whole reaction system was divided into less complex subsystems. For example, in order to analyze the formation of the pentamer AB separately, we employed a 5'aminodimer B', which was protected at its 3'-phosphate.

In a series of experiments, each subsystem was studied with respect to the effect of each reaction product. Standard oligodeoxynucleotides were employed as model templates. These experiments allowed us to decipher the dynamic structure of the whole system, which can be understood as a catalytic network, namely, an autocatalytic set with a total of six feedback couplings (Figure 4).

However, only those couplings with sufficient efficiency (denoted $(+)(+)$ and $(+)(+)(+))$ exert a notable influence. These strong couplings affect only the synthesis of the hexamer $\mathbf{A B C}$ and its pentameric precursor $\mathbf{A B}$. Both products are coupled autocatalysts: They behave as autocatalytic "egoists" and, at the same time, as mutually catalytic altruists. On the other hand, the tetramer AC which is formed as the main product via the non-auto-catalytic channel (G-G-stack leads to 
fast condensation), can be described as an isolated autocatalyst. The molecules $\mathbf{A B}$ and $\mathbf{A B C}$ compete with the main product $\mathbf{A C}$ for the incorporation of their common precursors $\mathbf{A}$ and $\mathbf{C}$. This competition was indeed observed: Upon the seeding of the reaction mixture with the hexameric template CCGCGG (ABC), the autocatalysts $\mathbf{A B}$ and $\mathbf{A B C}$ were formed more quickly, whereas the formation of the egoistic tetramer $\mathbf{A C}$ decreased. Competition of replicators for common resources is the prerequisite for selection. "Selection" in the biological sense usually means the takeover of resources by a species that reproduces more efficiently than its competitors (survival of the fittest). However, because selection also depends on the population level of a species (its concentration), a less efficient species may win if it starts at a higher population level. In any case, the population size of a species directs the flow of resource consumption. Although our experimental findings resemble selection, they represent only a rudimentary form of it: "True" Darwinian selection necessitates exponential, and not parabolic, growth.

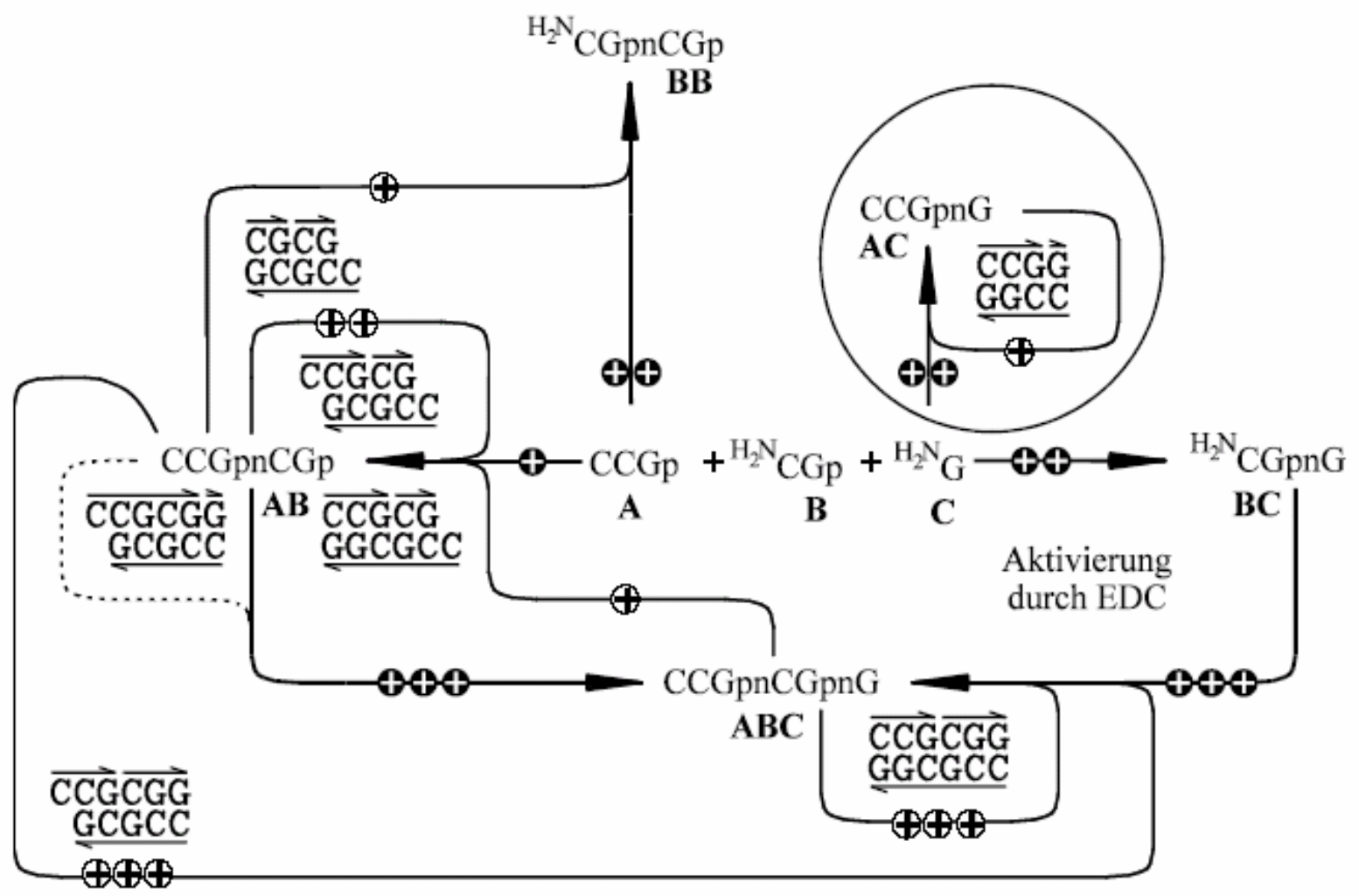

Figure 4. A self-replicating system with three starting materials.

All synthetic replicators described so far are based on the simplification of an autocatalytic, self-complementary system. However, the natural prototype of nucleic acid replication utilizes complementary, rather than self-complementary, strands (that is, replication via (+)- and (-)strands). The underlying principle of this type of replication is a cross-catalytic reaction in which 
one strand acts as a catalyst for the formation of the other strand and vice versa. It seemed worthwhile to test whether or not a replication of complementary hexadeoxynucleotides in the absence of any enzyme could be achieved. A minimal implementation of such a cross-catalytic self-replicating system can be represented by a simple reaction scheme (see Figure 5) where AA and $\mathbf{B B}$ denote templates, and $\mathbf{A}, \mathbf{A}^{\prime}, \mathbf{B}$, and $\mathbf{B}^{\prime}$ denote fragment molecules complementary to the templates.

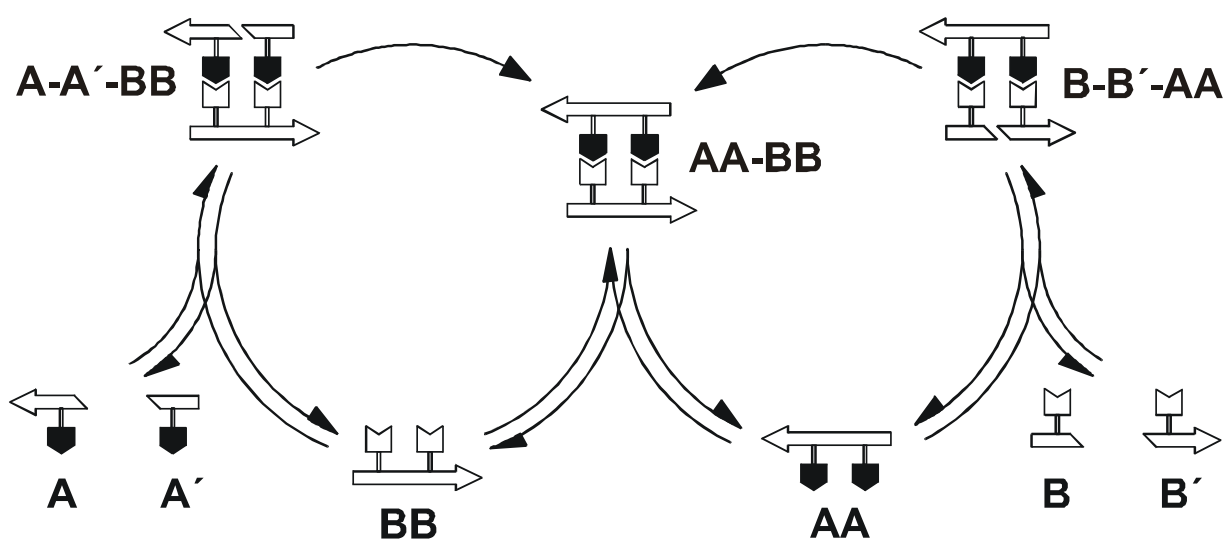

Figure 5. Minimal representation of a cross-catalytic self-replicating system.

In this scheme, two parallel pathways exist, both leading to the same template duplex. Since AA catalyzes the formation of $\mathbf{B B}$ and vice versa, one can speak of cross-catalysis. As long as both templates are formed using the same type of chemistry, the same conditions that enable cross-catalytic formation of complementary molecules $\mathbf{A A}$ and $\mathbf{B B}$ will also allow for the autocatalytic self-replication of both self-complementary products $\mathbf{A B}$ and $\mathbf{B A}$. For best observing the cross-catalytically coupled reactions, all four possible condensations between the precursor molecules must be equally efficient. Earlier experiments in our lab had shown that the efficiency of template-directed condensation reactions between suitably protected trideoxynucleotides was predominantly determined by the stacking of nucleobases flanking the reaction site within the termolecular complex. ${ }^{26}$ Accordingly, the cross-catalytic system could be realized experimentally by Sievers and von Kiedrowski et al. using the trimer precursors $\mathbf{A}$ (for $\mathrm{CCG}$ ) and $\mathbf{B}$ (for $\mathrm{CGG}$ ) to form a central GC sub-sequence in all four possible hexamers (AB, $\mathbf{B B}, \mathbf{A B}, \mathbf{B A}) .^{28,29}$ The formation of 3'-5' -phosphoramidate linkages was used to join the trimers. Kinetic analysis revealed that the two complementary sequences $\mathbf{A A}$ and $\mathbf{B B}$ are formed with similar efficiency, despite the difference in their pyrimidine content. When the experiment was performed in such a way that all products could form simultaneously, both the complementary and the self-complementary hexadeoxynucleotides exhibited the same time course of formation. In other words, cross-catalysis was as efficient as autocatalysis. On the contrary, in single experiments where only one hexamer could form, the autocatalysts were formed much faster than the complementary oligonucleotides. This is the expected result, since by definition only the 
autocatalyst is able to accelerate its own synthesis. Cross-catalysis, again by definition, necessitates the simultaneous formation of both complementary products and thus cannot be observed in single experiments in which only one product is formed. As expected, the crosscatalytic self-replication of complementary hexamers revealed parabolic growth characteristics due to product inhibition.

The general scheme of a minimal self-replicating system (Figure 1) has served as a successful aid for the design of various new replicators. In these systems, the rate of autocatalytic synthesis typically depends on the square root of the template concentration, and thus the template growth is parabolic rather than exponential. It was shown theoretically by Szathmáry and Gladkih ${ }^{12}$ that parabolic growth leads to the coexistence of self-replicating templates, which compete for common resources under stationary conditions. Coexistence in this context means that the faster replicator is not able to take over the common resources completely. If two nonself-replicating molecules, denoted $\mathrm{C}_{1}$ and $\mathrm{C}_{2}$, are competing for common precursors, the ratio of their concentrations is determined solely by their reactivity: $\left[\mathrm{C}_{1}\right] /\left[\mathrm{C}_{2}\right]=\mathrm{k}_{1} / \mathrm{k}_{2}$. The quotient $\left[\mathrm{C}_{1}\right] /\left[\mathrm{C}_{2}\right]$ describes selectivity. For two parabolic replicators with the autocatalytic rate constants $\mathrm{k}_{1}$ and $\mathrm{k}_{2}$, it follows from Szathmáry's treatment that $\left[\mathrm{C}_{1}\right] /\left[\mathrm{C}_{2}\right]=\left(\mathrm{k}_{1} / \mathrm{k}_{2}\right)^{2}$. Hence, small differences in reactivity lead to a higher selectivity in parabolic replicators as compared to nonself-replicating molecules. This enhancement of selectivity is partly implicit in the results reported.

It was pointed out by Szathmáry that the Darwinian kind of selection (survival of the fittest) necessitates exponential growth of competing replicators. For equilibrated self-replicating systems (in which the template-directed condensation is slow compared to internal equilibration), the autocatalytic reaction order (which is 1 in the case of exponential growth and $1 / 2$ in the case of parabolic growth) is determined solely by the population of the complexes involved. A minimal self-replicating system as presented in Figure 1 is expected to exhibit exponential growth if the termolecular complex is thermodynamically more stable than the template duplex (due to entropic reasons, the usual situation is just opposite to that).

Since exponential growth has been described as the prerequisite for selection in the Darwinian sense, experimental approaches aimed on the realization of molecular evolution via different strategies. One strategy is the introduction of a catalytically active covalently bound leaving group, a minimal replicase, which increases the thermodynamic stability of the termolecular complex. As long as the replicase is part of the termolecular complex, it stabilizes the latter, for example by wrapping itself around the complex. Stabilization occurs in an intramolecular sense.

Using the technique of directed molecular evolution Burmeister ${ }^{30}$ and Azzawi ${ }^{31}$ screened pools of random oligodeoxynucleotides to isolate molecules that are able to catalyze the EDCdriven self-replication of a self-complementary hexa- or decadeoxynucleotide. The goal was to find an oligodeoxynucleotide that would act as a nucleophilic catalyst of the phosphoryl transfer step occurring in the termolecular complex (covalent catalysis). The selection started with a synthetic pool of random oligodeoxynucleotides bearing 5'- and 3'-constant regions as primer 
binding sites for PCR amplification. A modified primer containing a 5'-terminal biotin and a 3'5'-phosphoramidate linkage following the leader sequence CCG (primer-trimer-conjugates) was synthesized. PCR amplification of the random pool using the modified 5'-primer, as well as a conventional 3'-primer, gave a pool of double-stranded DNA. Immobilization of the doublestranded pool on a streptavidin column and subsequent denaturation results in a single-stranded DNA pool containing one 3'-5'-phosphoramidate linkage. The in vitro selection was performed on the column in the presence of the attacking trimer (CGG) and the hexameric template (CCGCGG). The 5'-amino oligomers released during selection were eluted from the column. PCR amplification of the column eluate yielded the pool for the next round of selection. Successive rounds of kinetic selection and PCR

(CGG) and the hexameric template (CCGCGG). The 5'-amino oligomers released during selection were eluted from the column. PCR amplification of the column eluate yielded the pool for the next round of selection. Successive rounds of kinetic selection and PCR amplification were expected to result in selective enrichment of active sequences. From a DNA-pool containing 72 randomized positions embedded in two constant sequences two dominating sequences were isolated. Unfortunately, an examination of these sequences revealed that the oligonucleotides did not catalyze the desired ligation reaction but the hydrolysis of the internal phosphoramidate bond in the presence of the trinucleotide (CGG) as a cofactor.

An exponential non-enzymatic amplification of oligonucleotides was realized in our laboratory on the surface of a solid support in a stepwise replication procedure called SPREAD (Surface Promoted Replication and Exponential Amplification of DNA analogues). In the procedure described by Luther et al. in 1998 two template molecules were separately immobilized on solid support and hybridized with complementary oligonucleotide building blocks, which were ligated to the corresponding template molecules by EDC (Figure 6). ${ }^{32}$

The following separation and immobilization of the product molecules yields further material for another replication cycle. The oligonucleotides connected to the solid-phase via a disulfidebond were removed by reduction and analyzed by RP-HPLC. In this approach the duplex formation of the template was prevented by immobilization and therefore no product inhibition could occur. This iterative procedure is the first non-enzymatic exponential replication in a nonautonomous system.

All experiments on self-replication of oligonucleotides have been analyzed by RP-HPLC so far. Since this method is very laborious and only provides limited data it was necessary to develop rapid techniques for monitoring the chemical kinetics in a parallel fashion. From our group Schöneborn and Bülle (2001) reported on the online monitoring of chemical replication of self-complementary oligonucleotides by means of FRET (Fluorescence Resonance Energy Transfer). ${ }^{33}$ The chosen oligonucleotide sequences were based on the hexameric replication system described before which was extended to an octameric (TCCGCGGA) a decameric (TTCCGCGGAA) and a dodecameric (TTTCCGCGGAAA) system by elongating with dT and $\mathrm{dA}$. The two fluorescent dyes, donor $\mathrm{Cy} 3$ and acceptor $\mathrm{Cy} 5$ were introduced at the 5 '-end to give a labeled educt $\mathbf{A}$ and a labeled template molecule $\mathbf{C}$ (see Figure 7). Since the population of the 
termolecular complex is small the product formation in the template duplex $\mathbf{C}_{2}$ gives the desired FRET signal.

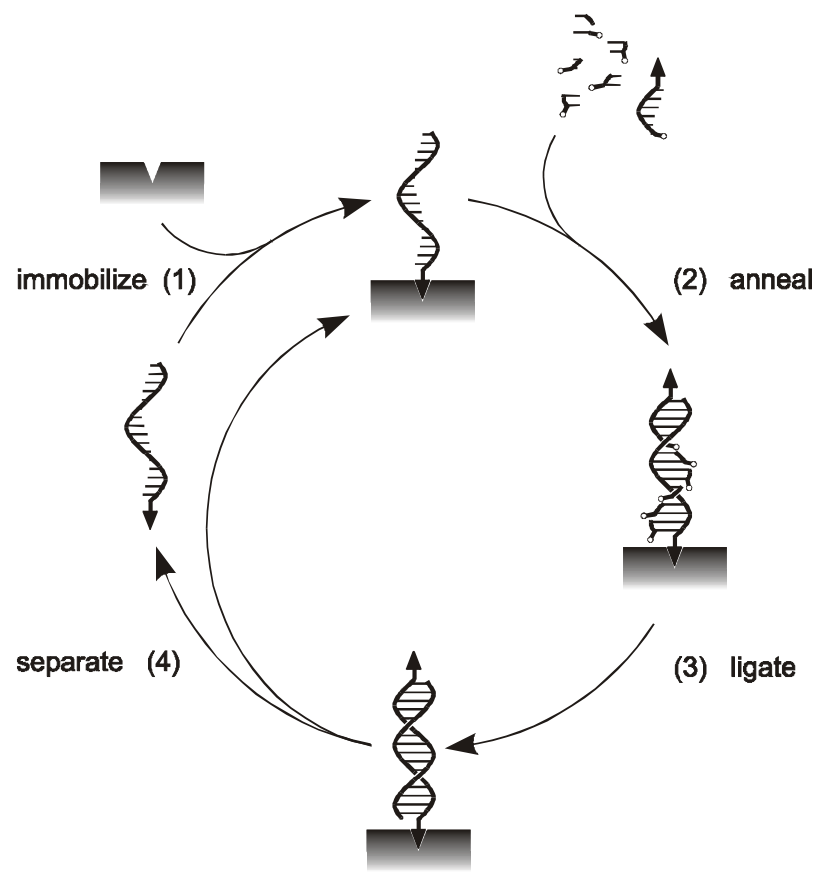

Figure 6. General scheme of the SPREAD procedure.

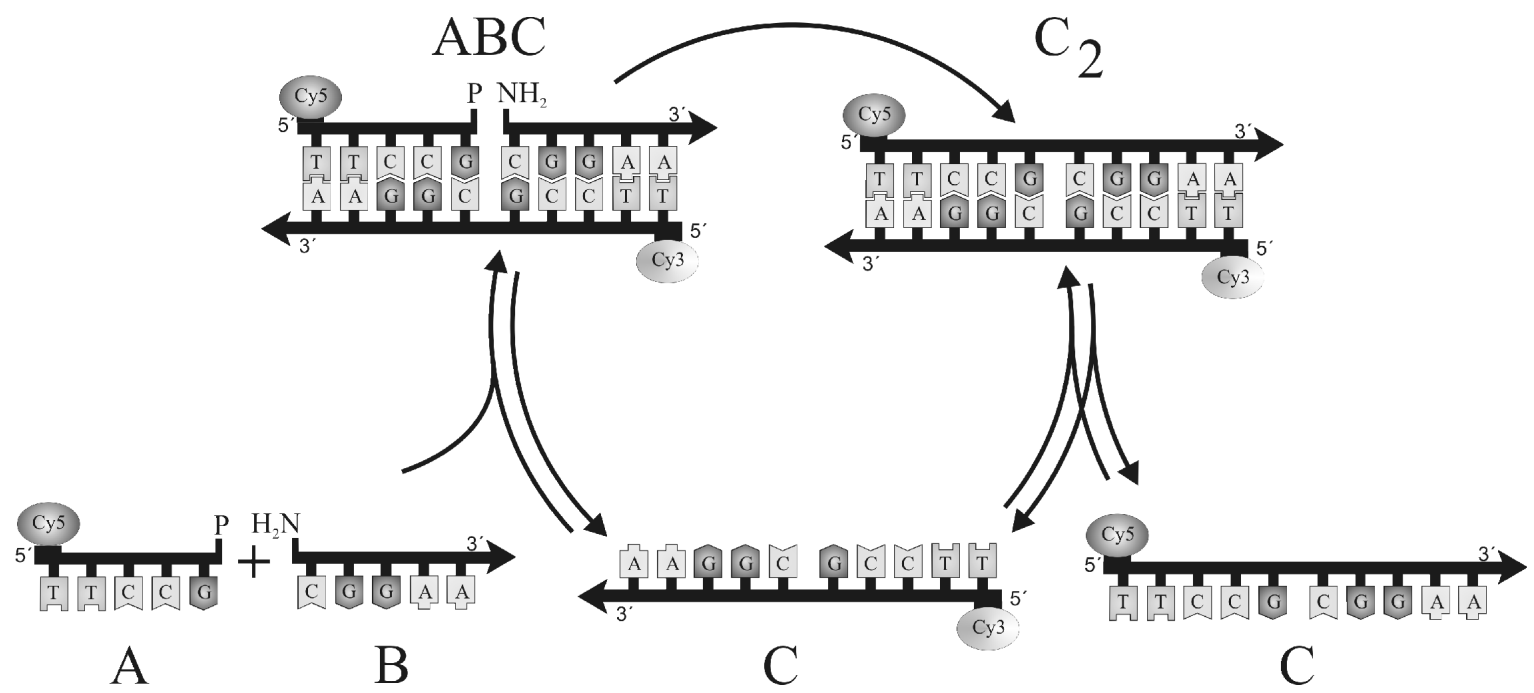

Figure 7. Scheme of a self-replicating system analyzed by FRET.

The chemical ligation in this system is achieved via EDC activation to yield a phosphoramidate product as described before. The FRET-based method allows a fast examination for a number of different self-replicating systems. As the experiments can also be 
carried out on microtiter plates it is a suitable technique for the rapid screening of factors that exhibit an influence on the dynamics of autocatalytic growth like a, "minimal replicase" for instance.

Further developments of oligonucleotide self-replicating systems aimed on the development of new ligation methods in order to avoid hydrolyzable condensing agents as EDC. Especially at high temperatures the formation of side products increases in the EDC driven ligation. A number of alternative ligation reactions proceeding without activating reagents were described in literature. Examples include the reaction of 3'-phosphorthioate- or 3'-phosphorselenoate compounds with $5^{\prime}$-iodine-substituted compounds, the formation of monophosphoryldisulfides from 3'-phosphorthioates with 5'-mercapto-oligonucleotides or the reaction of $3^{\prime}-$ or $5^{\prime}$ hydrazides with $3^{\prime}$ - or 5'-aldehydes.

In our group the formation of a $3^{\prime}-5^{\prime}$-disulfide bond via disulfide-exchange reactions was recently examined in an oligonucleotide self-replicating system. The reaction was monitored by UV-spectroscopy as characteristically UV-active leaving groups like Ellman's ion were used for thiol activation. ${ }^{34}$ Autocatalysis was proved for this system, but due to the very fast exchange reaction the non-catalytic reaction was predominant under the chosen conditions. Nevertheless, the disulfide ligation has some advantages compared to the phosphoramidate ligation, because no side products were formed and the reaction rate can be influenced by $\mathrm{pH}$-value. Also a variation or modification of the leaving group can be used to affect the reaction rate and form reactive and stable conjugates for the concept of, "minimal replicase".

\section{Replication in non-nucleotidic model systems}

Another exciting field in bioorganic chemistry is the development of non-nucleotidic replicators. Since molecular recognition and catalysis are common features among various organic molecules, it seems feasible to develop new replicators based on truly artificial precursors. Rebek and co-workers designed a replicator consisting of an adenosine derivative as the natural component and a derivative of Kemp's acid as the artificial part. ${ }^{35,36}$ 


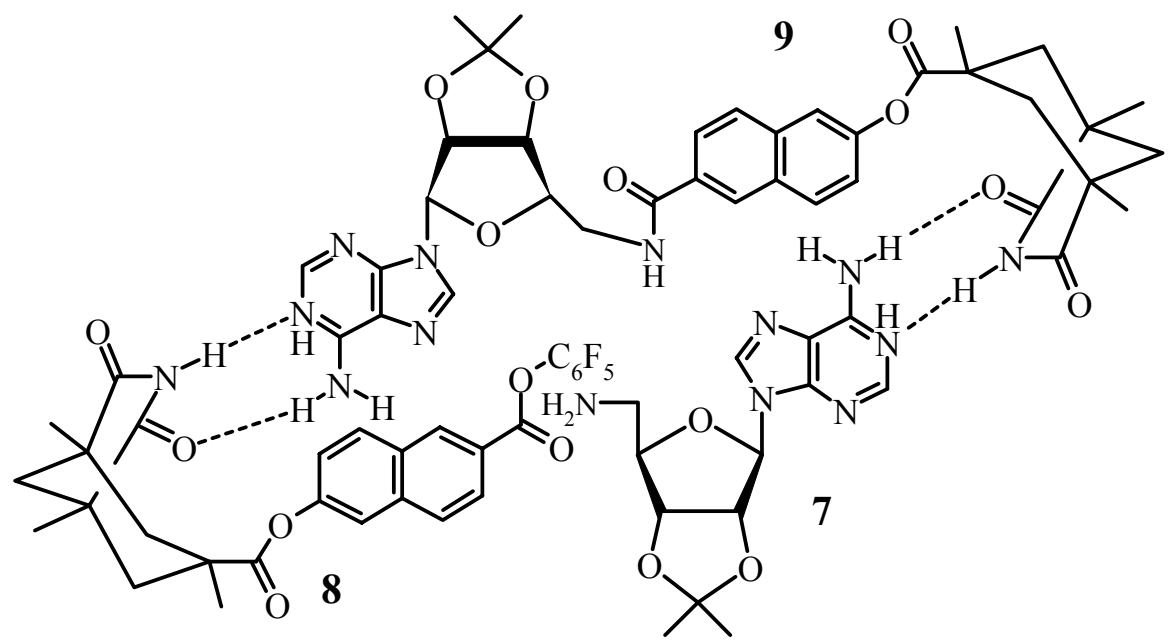

Figure 8. Synthetic self-replicating system according to Rebek. ${ }^{35,36}$

As shown in Figure 8, the reactive ends of the reactants 7 and 8 come into spatial proximity when the reactants interact with the template 9. Nucleophilic attack of the primary amine of 7 on the activated carboxyl ester of $\mathbf{8}$ leads to amide bond formation, giving a new template molecule of 9. Dissociation of the self-complementary template duplex closes the replication cycle. A close examination of the system revealed that also bimolecular pathways lead to product formation at high educt concentrations. ${ }^{37,38}$

Rebek's replicator challenged us to think about an even simpler self-replicating system. Terfort and von Kiedrowski used the condensation of 3-aminobenzamidine 10 and (2formylphenoxy)-acetic acid $\mathbf{1 1}$ to develop an artificial self-replicating system based on simple organic molecules. 39

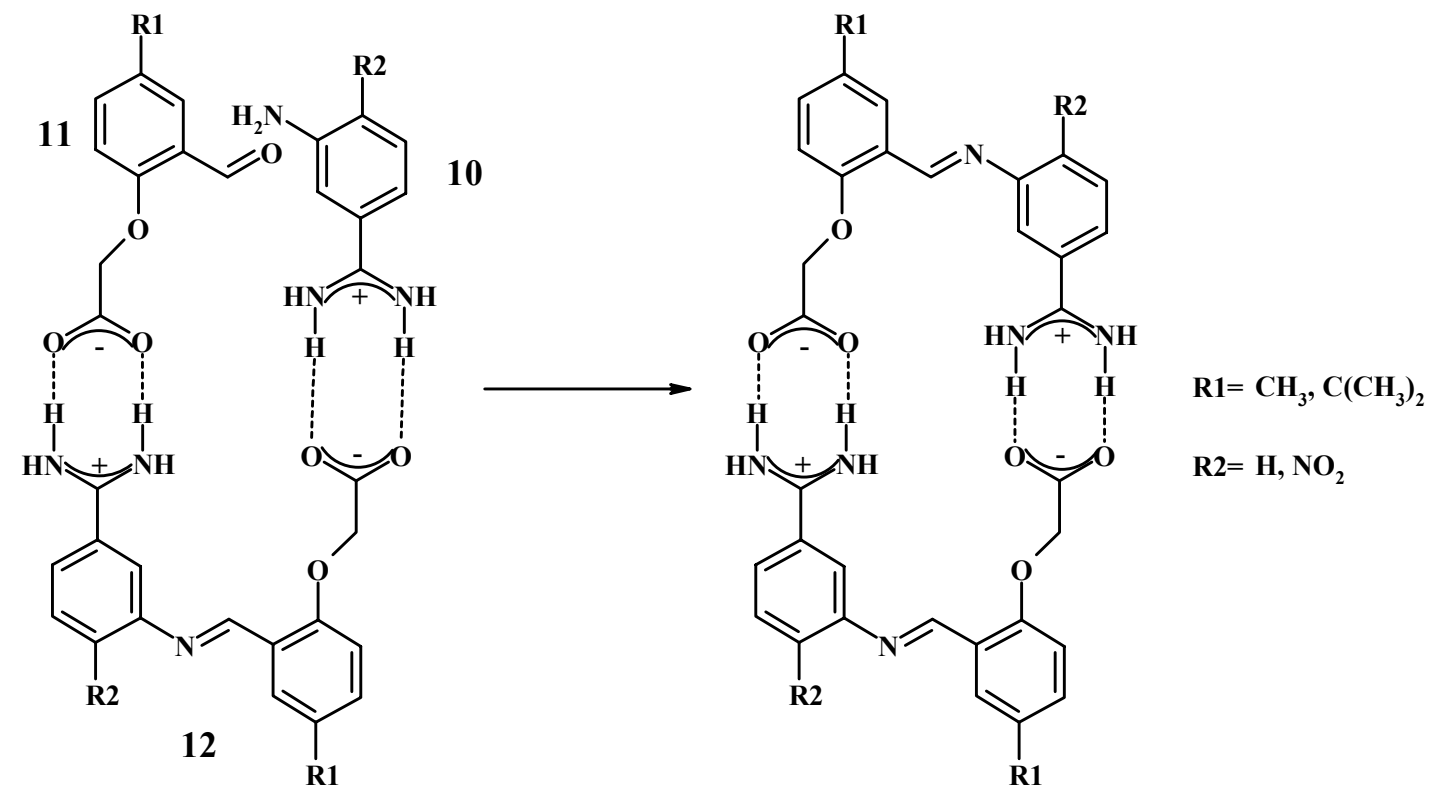

Figure 9. Self-replication of amidinium-carboxylate templates. 
As illustrated in Figure 9, autocatalytic condensation of $\mathbf{1 0}$ and $\mathbf{1 1}$ giving the anil $\mathbf{1 2}$ was followed by ${ }^{1} \mathrm{H}$ - NMR spectroscopy in dimethyl sulfoxide. As expected, the autocatalytic contribution of the condensation reaction shows a square-root law.

Another artificial self-replicating system was presented by Wang and Sutherland in $1997 .{ }^{40}$ Analysis revealed that the autocatalytic reaction order in this system lies between parabolic and exponential growth (reaction order of 0.8). The chemical ligation in this system is based on Diels-Alder-reaction between a cyclohexadiene derivative and an N-substituted maleimide as dienophile. A further increase of the autocatalytic reaction order to 0.89 was achieved by Kindermann and Stahl in our group, with another Diels-Alder-replicator. ${ }^{41}$ In these systems the product inhibition seems to be reduced, owing to the fact that the association of the template molecules is sterically hindered, whereas the close spatial proximity in the termolecular complex is suitable for ligation. By this stabilization of the termolecular complex compared to the template duplex the observed reaction order follows the theoretical requirements for exponential growth. The minimal self-replicating system presented by Philp in 2001 is based on a 1,3-dipolar cycloaddition between a nitrone and a maleimide. ${ }^{42}$ Here, only one of the two diastereoisomeric products is capable of acting as an efficient template for its own formation. The autocatalytic reaction order was determined with 0.9 due to a destabilisation of the template duplex.

In 1996 Lee and Ghadiri et al. demonstrated that non-enzymatic self-replication can also be realized based on peptides. ${ }^{43}$ The described system is based on a 32- amino acid peptide with an $\alpha$-helical coiled-coil-structure of repetitive 7 mer units (abcdefg) . The reacting fragments were a $17 \mathrm{mer}$ as electrophile and a $15 \mathrm{mer}$ as the nucleophile which form an amide bond via Kent ligation. The molecular recognition is based on hydrophobic (position a and d) and electrostatic (position e and g) interactions in the coiled-coil (see Figure 10). The kinetic data from the system was gained by RP-HPLC analysis and examination of the system revealed parabolic growth as expected. Interestingly at higher starting concentrations of peptides an increase in autocatalytic growth was observed (reaction order of 0.63). As the reaction order is increasing with the number of templates involved in the complex this results were explained by the formation of a quaternary complex ABTT which leads to a termolecular product complex TTT.
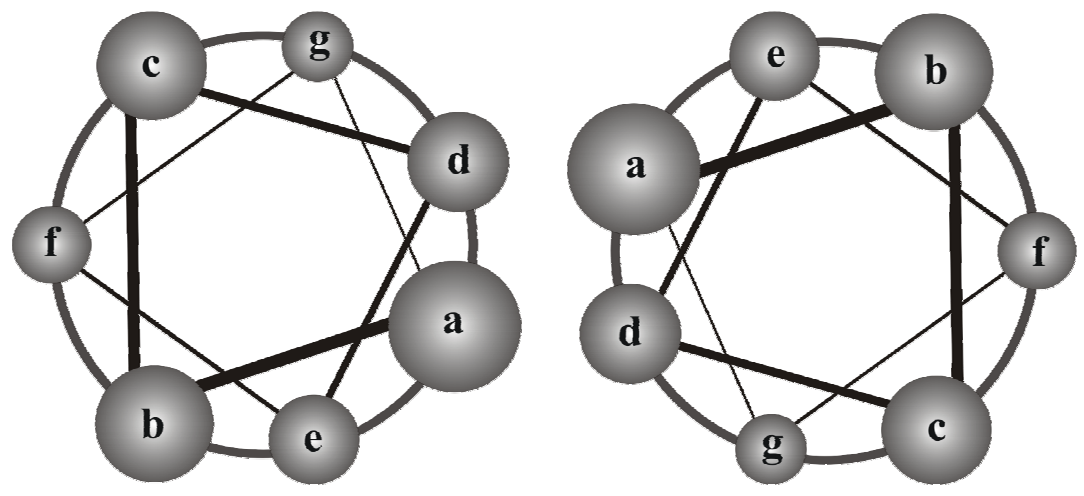

Figure 10. Schematic view of a self-replicating peptide system. 
A further development of the self-replicating peptides towards exponential growth was also achieved by a destabilization of the template duplex. Chmielewsiki et al. reported on two replicators with a very high reaction order of 0.91 . In one case, in 2001, this was achieved by shortening the coiled coil to its minimum length of 26 amino acids. ${ }^{44}$ In the other case (2003) the insertion of one proline amino acid in the building blocks of the replicating peptides leads to a nick which destabilizes the template duplex. ${ }^{45}$

\section{Outlook: toward exponential replication}

It was pointed out by Szathmáry that the Darwinian kind of selection (survival of the fittest) necessitates exponential growth of competing replicators. For equilibrated self-replicating systems (in which the template-directed condensation is slow compared to internal equilibration of the molecule complexes), the autocatalytic reaction order is determined solely by the population of the complexes involved. A minimal self-replicating system presented in Figure 1 is expected to exhibit exponential growth if the termolecular complex is thermodynamically more stable than the template duplex.

These theoretical considerations have to some extent been proven in synthetic replicators, where high reaction orders were observed for adequately designed systems with destabilized product duplexes. To overcome product inhibition in minimal self-replicating systems of oligonucleotides the destabilization of template duplexes might be practicable by a change in ligation chemistry or performing the template replication at surfaces. Using the new techniques for analyzing and ligating oligonucleotides the search for a, "minimal replicase" is still a promising concept to overcome product inhibition. This concept can even be extended to the concept of an artificial cell, where the leaving group is a stabilizing lipid which can transport the oligonucleotide through cell membranes and form new membranes after cleavage.

\section{References}

1. Oparin, A. I. In The Origin of Life; Pabochii: Moscow, 1924.

2. Kühn, H., Waser, J. Angew. Chem. 1981, 93, 495.

3. Crick, F. H. C. J. Mol. Biol. 1968, 38, 367.

4. Orgel, L. E. J. Mol. Biol. 1968, 38, 381.

5. Eigen, M.; Schuster, P. The Hypercycle: A Principle of Natural Self-Organisation, Springer: Berlin, 1979.

6. Kühn, H.; Waser, J. Angew. Chem. 1981, 93, 495.

7. Joyce, G. F. Nature 1989, 338, 217.

8. Sharp, P. A. Cell 1985, 42, 397.

9. Cech, T. R. Proc. Natl. Acad. Sci. USA 1986, 83, 4360. 
10. Breaker, R.; Joyce, G. F. Chemistry and Biology 1994, 1, 223.

11. Paul, N.; Joyce, F. G. Proc. Natl. Acad. Sci. USA 2002, 99, 12733.

12. Szathmáry, E.; Gladkih, I., J. Theor. Biol. 1989, 138, 55.

13. Orgel, L. E.; Lohrmann, R. Acc. Chem. Res. 1974, 7, 368.

14. Inoue, T.; Orgel, L. E. Science 1983, $219,859$.

15. Orgel, L. E. Nature 1992, 358, 203.

16. Naylor, R.; Gilham, P. T. Biochemistry 1966, 5, 2722.

17. Dolinnaya, N. G.; Sokolova, N. I.; Gryaznova O. L.; Shabarova Z. A. Nucleic Acids Res. 1988, 16, 3721.

18. Dolinnaya, N. G.; Tsytovich, A. V.; Sergeev, V. N.; Oretskaya, T. S.; Shabarova, Z. A. Nucleic Acids Res. 1991, 19, 3073.

19. von Kiedrowski, G. Angew. Chem. Int. Ed.1986, 25, 932.

20. Zielinski, W. S.; Orgel, L. E. Nature 1987, 327, 346.

21. von Kiedrowski, G. Bioorg. Chem. Front. 1993, 3, 113.

22. von Kiedrowski, G.; Wlotzka, B.; Helbing, J. Angew. Chem. Int. Ed. 1989, 28, 1235.

23. von Kiedrowski, G. Selbstreplikation in Chemischen Minimalsystemen. 40 Jahre Fonds der Chemischen Industrie 1950-1990. VCI: Frankfurt 1990, 197.

24. von Kiedrowski, G., Wlotzka, B., Helbing, J., Matzen, M.; Jordan, S., Angew. Chem. Int. Ed. 1991, 30, 423; and corrigendum p 892.

25. Rotello, V.; Hong, J. L.; Rebek, J. J. Am. Chem. Soc. 1991, 113, 9422.

26. Wlotzka, B. Ph.D. Thesis, University of Göttingen, 1992.

27. Achilles, T.; von Kiedrowski, G. Angew. Chem. Int. Ed. 1993, 32, 1198.

28. Sievers, D.; von Kiedrowski, G. Nature 1994, 369, 221. Sievers, D., v. Kiedrowski, G. Chem. Eur. J. 1998, 629.

29. Sievers, D.; Achilles, T.; Burmeister, J.; Jordan, S.; Terfort, A.; von Kiedrowski, G., Ed. G. R. Fleischaker, 1994, pp 45.

30. Burmeister, J.; von Kiedrowski, G.; Ellington, A. Angew. Chem. Int. Ed. 1997, 36, 1321.

31. Azzawi, A. Ph. D. Thesis, Ruhr-Universität-Bochum, 2001.

32. Luther, A.; Brandsch, R.; v. Kiedrowski, G. Nature 1998, 396, 245.

33. Schöneborn, H.; Bülle, J.; von Kiedrowski, G. Chembiochem. 2001, 12, 922.

34. Patzke, V. Ph. D. Thesis, Ruhr-Universität-Bochum, 2005.

35. Tjivikua, T.; Baluster, R.; Rebek, J. J. Am. Chem. Soc. 1990, 112, 1249.

36. Nowick, J. S.; Feng, Q.; Tjivikua, T.; Ballester, P.; Rebek, J. J. Am. Chem. Soc. 1991, 113, 8831 .

37. Menger, F. M.; Eliseev, A. V.; Khanjin, N. A. J. Am. Chem. Soc. 1994, 116, 3613.

38. Reinhoudt, D. N.; Rudkevic, D. M.; de Jong, F. J. Am. Chem. Soc. 1996, 118, 6880.

39. Terfort, A.; von Kiedrowski, G. Angew. Chem. Int. Ed. 1992, 31, 654.

40. Wang, B.; Sutherland, I. O. Chem. Comm. 1997, 1495.

41. Kindermann, M.; Stahl, I.; Reimold, M.; Pankau, W. M.; von Kiedrowski, G. Angew. Chem. Int. Ed. 2005, 44(41), 6750. 
42. Allen, V. C., Philp, D., Spencer, N. Org. Lett. 2001, 3, 777.

43. Lee D. H.; Granja J. R.; Martinez J. A.; Severin K.; Ghadiri, M. R. Nature 1996, 382, 525.

44. Issac, R.; Chmielewski, J. J. Am. Chem. Soc. 2002, 124, 6808.

45. Li. X.; Chmielewski, J. J. Am. Chem. Soc. 2003, 125, 11820. 